\title{
The comparison of the efficacy of swine FMD vaccine emulsified with oil adjuvant of ISA 201 VG or ISA 206 VG
}

\author{
Dong Li*, Chunxue Zhou, Daliang She, Pinghua Li, Pu Sun, Xingwen Bai, Yingli Chen, Baoxia Xie, \\ Zaixin Liu*
}

State Key Laboratory of Veterinary Etiological Biology, Key Laboratory of Animal Virology of Ministry of Agriculture, OIE/National Foot and Mouth Disease reference laboratory of China, Lanzhou Veterinary Research Institute, Chinese Academy of Agricultural Sciences, Lanzhou, China

Email: lidong@caas.cn,

Received September 2013

\begin{abstract}
The Seppic Company developed a new adjuvant Montanide ISA 201 VG, the upgraded version of Montanide ISA 206 VG, which keep the advantage and added some chemical components on the basis of ISA 206 to improve the cellular responses. The aim of the study is to compare the efficacy of swine FMD (foot-and-mouth) vaccine emulsified with oil adjuvant of ISA 201 or ISA 206 respectively. The pigs were vaccinated with $F M D$ vaccine emulsified with inactive FMD type $O$ antigen and adjuvant ISA 201 or ISA 206 respectively, according to $2.0 \mathrm{ml}$ (1/1 dose), 0.67 $\mathrm{ml}(1 / 3 \mathrm{dose}), 0.22 \mathrm{ml} \mathrm{(1/9}$ dose $)$ to calculate their PD50. The sera were collected from the vaccination of the day $0,3,7,14,21,28$ and the ELISA FMD type $O$ antibody were detected. Furthermore, the PD50 were calculated after the pigs were challenged with virulent FMDV type $O$ on 28 days post vaccination. The ELISA antibody titers of 201vaccine were significantly higher than that of 206 (except the third time). The fifty percent of protection dose (PD50) of 201 vaccine $(P D 50=15.59)$ was higher than that of 206 vaccine $(P D 50=10.05)$. The above data showed that the efficacy of the FMD vaccine emulsified with ISA 201 was better than which with ISA 206.
\end{abstract}

Keywords: Swine FMD Vaccine; Adjuvant; ISA 201; ISA 206; Immune Efficacy

\section{INTRODUCTION}

Foot-and-mouth disease (FMD is an acute, febrile, and contagious vesicular disease affecting cloven-hoofed animals. The causal agent, FMD virus (FMDV), is a member of the genus Aphthovirus in the family Picornaviridae and occurs as seven distinct serotypes throughout the

${ }^{*}$ Corresponding authors. world: A, O, C, Asia1 and South African Territories (SAT) 1-3 [1-3]. Vaccination is the most important control and eradication strategy for FMD, especially the oil-adjuvant vaccine in developing countries. Significant advances in recent years have led to the introduction of "ready-toformulate” oil adjuvants. Montanide ISA 206, the mineral based oil, which readily forms water-in-oil-in-water emulsion. Its efficacy in eliciting immune response was studied in FMD vaccines against FMDV [4-7].

The advantage of oil adjuvant was attributed to depot formation at the site of injection, a vehicle for transport of the antigen throughout the lymphatic system and slow antigen release with the stimulation of antibody producing cells. Moreover, being oil emulsion, Montanide ISA 206 had various advantages, like low viscosity, easy administration, longer term protection, greater stability and production of smaller nodules at the site of injection [5], compared to other oil adjuvants, making as an ideal adjuvant candidate for FMD vaccines. It was also suggested that Montanide ISA 206 could prevent the loss of potency was due to the proteolysis of VP1 or possibly the physical breakdown of the virus followed adsorption to the aluminum hydroxide gel [8] and agree with the usage of Montanide ISA 206 ready to formulate oil adjuvant can be sued in all target species is ideal for emergency vaccination [9]. The usage of oil adjuvant (Montanide ISA 206) improve, enhance cell mediated immunity and give higher level and long lasting immunity $[10,11]$. Other researchers [12,13] detected that DOE vaccine containing Montanide ISA 206 is highly efficient, fluidy with low viscosity which is easily dispersed from the place of injection and gave high $\mathrm{Ab}$ titers and longest duration of immunity than alhdyragel vaccine and Daoud reported that the duration of immunity elicited by gel FMD vaccine was short lived and antibody concentrations rapidly fall after administration, while oils adjuventated FMD vaccines gave a longer duration of immunity and suggested that the oil adjuvenated vaccines 
had potential as an alternative to the conventional aluminum hydroxide FMD vaccine [14]. Moreover Patil reported that the oil adjuvant elicited a better immune response at any time than did aluminum hydroxide gel FMD quadrivalent vaccines in goats, and the response developed quicker. Local tissue reactions such as granulomas and cysts to oil-adjuvants have been not detected [15].

Now the Seppic developed a new adjuvant Montanide ISA 201, the upgraded version of Montanide ISA 206, which keep the advantage and added some chemical components on the basis of ISA 206 to improve the cellular responses. The aim of the study is to compare the efficacy and safety of swine FMD vaccine emulsified with oil adjuvant of ISA 201 or ISA 206 respectively.

\section{MATERIALS \& METHODS}

\subsection{The Animals}

Fifty 2-month-old pigs, sero-negative for FMDV type O antibodies (titre 1:4 by LPB-ELISA) were randomly divided into four groups. 45 pigs in group 1-3 and 15 pigs in each group. The vaccine with ISA 206 was the first group. The vaccine with ISA 201 was the second group. The vaccine with PBS as the adjuvant was the third group. Three small groups (Five pigs in it) were divided in each group for three vaccine dose $2.0 \mathrm{ml}$ (1/1 full dose), $0.67 \mathrm{ml}$ ( $1 / 3$ full dose), $0.22 \mathrm{ml}$ (1/9 full dose) to determine the PD50 (protective dose 50\%). The left five pigs were in the 4th group as the control. All the pigs were bred in the $\mathrm{P} 3$ animal laboratory respectively.

\subsection{Vaccine Preparation}

The FMDV type $\mathrm{O}$ antigen was inactivated and safety checked by conventional method, and emulsified with ISA 201 or ISA 206 separately according to the prospectus guide. The vaccine was stored at $4^{\circ} \mathrm{C}$.

\subsection{Vaccination, Sera Collection and Virus Challenge}

Three types of FMD vaccines were intramuscular inoculated at the ear-root-neck area of 45 pigs with $2 \mathrm{ml}$, $0.67 \mathrm{ml}, 0.22 \mathrm{ml}$ inactivated vaccine respectively, and five pigs were bred without vaccination as negative control. Blood and sera samples were collected at $0,3,7,14$, 21, $28 \mathrm{dpv}$ (days post vaccination) to assay the FMDV type $\mathrm{O}$ antibody by LPB-ELISA. To demonstrate vaccine efficacy, all 50 pigs were challenged intramuscularly with 1000 PID 50/2 ml of FMDV type O suckling mice passaged strain at the ear-root-neck area after $28 \mathrm{dpv}$ and FMD symptoms were monitored for 10 days, and the PD50 was calculated with Karber method.

\section{THE RESULTS}

Define abbreviations and acronyms the first time they are used in the text, even after they have been defined in the abstract. Abbreviations such as IEEE, SI, MKS, CGS, sc, dc, and rms do not have to be defined. Do not use abbreviations in the title or heads unless they are unavoidable.

\subsection{The ELISA Antibody Titer of FMDV Type $O$ after Pigs Vaccinated}

The ELISA antibody titer of 201-vaccine (1/1 dose) was 186.12 on 21st dpv, 201.82 on 28th dpv, much higher than which of 206-titer $(\mathrm{p}<0.05)$, because the titer of 206-vaccine (1/1 dose) was 69.76 on 21st dpv, 57.28 on 28th dpv. The ELISA antibody titer of 201-vaccine (1/3 dose) was 133.48 on 21st dpv, 120.62 on 28th dpv, much higher than which of 206-titer $\mathrm{r}(\mathrm{p}<0.05)$, because the titer of 206-vaccine (1/3 dose) was 69.3 on 21st dpv, 71.96 on 28th dpv. The ELISA antibody titer of 201vaccine ( $1 / 9$ dose) was 65.54 on 21 st dpv, 84.36 on 28th dpv, much higher than which of 206-titer $r(p<0.05)$, because the titer of 206-vaccine (1/9 dose) was 30.9 on 21st dpv, 29.56 on 28th dpv (Figure 1). These data showed that the effect of 201-vaccine is better than 206vaccine.

\subsection{The FMD Virulent Virus Challenge to Immunized Pigs on 28dpv}

The challenge result was presented in Table 1. Fifty pigs were all protected in 201-vaccine group (PD50-201 = 15.59). Thirteen pigs were protected in 206-vaccine group (2 pigs had clinic symptom with 1/9 dose, PD50$206=10.05)$. Four pigs were protected in PBS-vaccine group (5 pigs had clinic symptom with $1 / 9$ dose, 3 pigs had clinic symptom with $1 / 3$ dose, 3 pigs had clinic symptom with $1 / 1$ dose, PD50-PBS = 1.39). Generally, the FMD vaccine is qualified when PD50 comes up to 6.0. The above data showed that the PD50 of 201 emulsified FMD vaccine was higher than which of 206 emulsified vaccine.

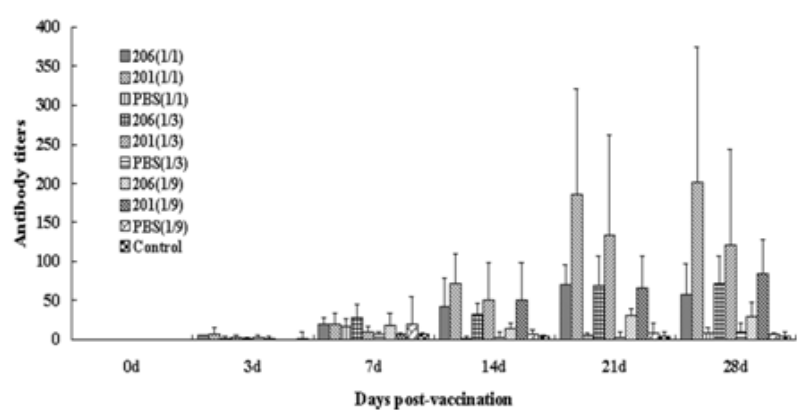

Figure 1. Comparisons of the ELISA antibody titers among 201-vaccine, 206-vaccine, PBS-vaccine. 
Table 1. The FMD clinical incidence after challenge.

\begin{tabular}{ccccccccccccc}
\hline Groups & & \multicolumn{7}{c}{ Pig No \& clinical incidence } \\
\hline $\mathbf{2 0 6}(\mathbf{1} / \mathbf{1})$ & 3051 & $(-)$ & 3056 & $(-)$ & 3057 & $(-)$ & 3076 & $(-)$ & 3098 & $(-)$ \\
$\mathbf{2 0 6}(\mathbf{1} / \mathbf{3})$ & 4441 & $(-)$ & 3074 & $(-)$ & 3047 & $(-)$ & 3075 & $(-)$ & 3077 & $(-)$ \\
$\mathbf{2 0 6}(\mathbf{1} / \mathbf{9})$ & 3072 & $(-)$ & 3060 & $(+)$ & 3063 & $(-)$ & 3065 & $(+)$ & 3083 & $(-)$ \\
$\mathbf{2 0 1}(\mathbf{1} / \mathbf{1})$ & 3058 & $(-)$ & 3059 & $(-)$ & 4477 & $(-)$ & 3079 & $(-)$ & 3086 & $(-)$ & $(-)$ \\
$\mathbf{2 0 1}(\mathbf{1} / \mathbf{3})$ & 3097 & $(-)$ & 3073 & $(-)$ & 3094 & $(-)$ & 3082 & $(-)$ & 3043 & $(-)$ & $(-)$ \\
$\mathbf{2 0 1}(\mathbf{1} / \mathbf{9})$ & 3064 & $(-)$ & 3092 & $(-)$ & 3081 & $(-)$ & 3070 & $(-)$ & 3069 & $(-)$ \\
PBS (1/1) & 3055 & $(-)$ & 3067 & $(-)$ & 3093 & $(+)$ & 3084 & $(+)$ & 3089 & $(+)$ \\
PBS (1/3) & 3054 & $(-)$ & 3061 & $(+)$ & 3095 & $(+)$ & 3068 & $(-)$ & 3088 & $(+)$ \\
PBS (1/9) & 3044 & $(+)$ & 3090 & $(+)$ & 3087 & $(+)$ & 3085 & $(+)$ & 3096 & $(+)$ \\
Control & 3099 & $(+)$ & 1040 & $(+)$ & 1439 & $(+)$ & 3080 & $(+)$ & 3071 & $(+)$ \\
\hline
\end{tabular}

Note: (+) means FMD clinic sighs appeared.

\section{DISCUSS}

The ISA206 adjuvant has applied in FMD vaccine production and other vaccines for 20 years, because it has lower side-reactions and viscosity, easier to emulsify and injection, and higher stability. Recently, The ISA 201 has developed on the base of ISA 206, which inherited advantages of ISA 206, and added some chemical substances with the hope of enhancing animals' cellular immune reactions [16]. In this report, the comparisons between ISA 201 and ISA 206 were done as the FMD vaccine respectively.

First the ELISA antibodies against FMDV type $\mathrm{O}$ were compared. The antibody titer induced by 201-vaccine were higher than which of 206-vaccine on 3dpv, $7 \mathrm{dpv}, 14 \mathrm{dpv}, 21 \mathrm{dpv}, 28 \mathrm{dpv}$. This means that the effect of 201-vaccine in inducing antibody is better than which of 206-vaccine.

The virus challenge trial to vaccinated animals is a very important index for evaluating the potency of FMD vaccine, which is indicated with PD50 (50 percent protection dose) $[17,18]$. Here, fifty pigs were all protected immunized with 201 vaccine, but thirty pigs were protected with 206 vaccine, which means that the potency of 201 vaccine is better than that of 206 vaccine.

\section{CONCLUSION}

From above, the efficacy of the FMD vaccine emulsified with ISA 201 is superior to ISA 206.

\section{ACKNOWLEDGEMENTS}

This work has been supported by "Seppic, France”.

\section{REFERENCES}

[1] Reuckert, R.R. (1996) Picornaviridae: the viruses and their replication. In: Fields, B.N., Knipe, D.M. and How- ley, P.M. Eds., Fields Virology, Lippincott-Raven, Philadelphia, 609-654.

[2] Doel, T.R. (2003) FMD vaccines. Virus Research, 91, 8199. http://dx.doi.org/10.1016/S0168-1702(02)00261-7

[3] Ward, M.P., Laffan, S.W. and Highfield, L.D. (2007) The potential role of wild and feral animals as reservoirs of foot-and-mouth disease. Preventive Veterinary Medicine, 80, 9-23.

http://dx.doi.org/10.1016/S0168-1702(02)00261-7

[4] Doel, T.R., Williams, L. and Barnet, P. (1994) Emergency vaccination against foot-and-mouth disease: Rate of development of immunity and its implications for the carrier state. Vaccine, 12, 592-600.

http://dx.doi.org/10.1016/0264-410X(94)90262-3

[5] Barnett, P.V., Pullen, L., Williams, L. and Doel T.R. (1996) International bank for foot-and-mouth disease: Assessment of Montanide ISA 25 and ISA 206, two commercially available oil adjuvants. Vaccine, 14, 11871198. http://dx.doi.org/10.1016/S0264-410X(96)00055-2

[6] Hunter, P. (1996) The performance of southern African territories serotypes of foot and mouth disease antigen in oil-adjuvanted vaccines. Revue Scientifique et Technique, 15, 913-922.

[7] Cox, S.J., Barnett, P.V., Dani, P. and Salt J.S. (1999) Emergency vaccination of sheep against foot-and-mouth disease: Protection against disease and reduction in contact transmission. Vaccine, 17, 1858-1868. http://dx.doi.org/10.1016/S0264-410X(98)00486-1

[8] Doel, T.R. and Pullen, L. (1990) International bank for foot-and-mouth disease vaccine: stability studies with virus concentrates and vaccines prepared from them. Vaccine, 8, 473-478. http://dx.doi.org/10.1016/0264-410X(90)90249-L

[9] Barnett, P.V. and Carabin, H. (2002) A review of emergency foot-and-mouth disease (FMD) vaccines. Vaccine, 20, 1505-1514. http://dx.doi.org/10.1016/S0264-410X(01)00503-5

[10] Sonia, A.M. (2007) Studies on preparation improved FMD virus oil adjuvant vaccine. VMVSc Thesis (Virology), University of Cairo, Cairo. 
[11] Fatthia, A.M. (2003) Vaccination of goats with FMD vaccines. VMVSc Thesis (Infectious Disease), University of Alexandria, Alexandria.

[12] Mohamed, A.A. (1998) Study on new double oil adjuvant for FMD virus vaccine. VMVSc Thesis (Virology), University of Cairo, Cairo.

[13] Wafaa, E.H. (1999) Epizootiological and immunological Studies on Foot and Mouth Disease. Ph.D. Thesis (Infectious Disease), University of Cairo, Cairo.

[14] Daoud, A.M., Ali, S.M. and Yousef, M.R. (2002) Comparative study between different new oil adjuvants used for production of FMD vaccine in sheep. 4th International Science Conference, 667-673.

[15] Patil, P.K., Bayry, J., Ramakrishna, C., Hugar, B., Misra,
L.D. and Prabhudas, K. (2002) Immune responses of sheep to quadrivalent double emulsion foot-and-mouth disease vaccines: Rate of development of immunity and variations among other ruminants. Journal of Clinical Microbiology, 40, 4367-4371. http://dx.doi.org/10.1016/S0264-410X(01)00503-5

[16] http://www.seppic.com/animal-health/vaccine-adjuvant/ montanide-isa

[17] http://www.oie.int/

[18] Li, D., Lu, Z.J., Xie, B.X., Sun, P., Chen, Y.L., Fu, Y.F. and Liu, Z.X. (2010) Alternative way to test the efficacy of swine FMD vaccines: Regulation of live virus challenge dose. Virology Journal, 7, 215.

http://dx.doi.org/10.1186/1743-422X-7-215 